\title{
Mathematical analogies in physics. Thin-layer wave theory
}

\author{
José M. Carcione ${ }^{1, \star}$, Vivian Grünhut ${ }^{2}$, Ana Osella ${ }^{2}$ \\ ${ }^{1}$ Istituto Nazionale di Oceanografia e di Geofisica Sperimentale (OGS), Sgonico (Trieste), Italy \\ ${ }^{2}$ Universidad de Buenos Aires, Departamento de Física - IFIBA Conicet, Buenos Aires, Argentina
}

\author{
Article history \\ Received April 8, 2013; accepted October 14, 2013. \\ Subject classification: \\ Thin layer, Electromagnetism, Viscoelasticity, Reflection and transmission coefficient, Geophysics, Quantum mechanics.
}

\begin{abstract}
Field theory applies to elastodynamics, electromagnetism, quantum mechanics, gravitation and other similar fields of physics, where the basic equations describing the phenomenon are based on constitutive relations and balance equations. For instance, in elastodynamics, these are the stress-strain relations and the equations of momentum conservation (Euler-Newton law). In these cases, the same mathematical theory can be used, by establishing appropriate mathematical equivalences (or analogies) between material properties and field variables. For instance, the wave equation and the related mathematical developments can be used to describe anelastic and electromagnetic wave propagation, and are extensively used in quantum mechanics. In this work, we obtain the mathematical analogy for the reflection/refraction (transmission) problem of a thin layer embedded between dissimilar media, considering the presence of anisotropy and attenuation/viscosity in the viscoelastic case, conductivity in the electromagnetic case and a potential barrier in quantum physics (the tunnel effect). The analogy is mainly illustrated with geophysical examples of propagation of $S$ (shear), $P$ (compressional), TM (transverse-magnetic) and TE (transverse-electric) waves. The tunnel effect is obtained as a special case of viscoelastic waves at normal incidence.
\end{abstract}

\section{Introduction}

The 19th century was a period when scientists had a global vision of science, making wide use of analogies between different field of physics, in particular the analogy between light and elastic waves to study the behaviour of light in matter. For instance, Fresnel's formulae and Maxwell's equations were obtained from mathematical analogies with shear wave propagation and Hooke's law, respectively [e.g., Carcione and Cavallini 1995, Carcione and Robinson 2002]. This practice dates back to the 17 th century. Hooke believed light to be a vibratory displacement of a medium (the ether), through which it propagates at finite speed.

The role of mathematical analogies has been well illustrated and explained by Tonti in a series of papers [e.g., Tonti 1972, 1976]. Quoting Tonti [1972]: "Many physical theories show formal similarities due to the existence of a common mathematical structure. This structure is independent of the physical contents of the theory and can be found in classical, relativistic and quantum theories; for discrete and continuous systems". Carcione and Cavallini [1995] found analogies between vector wave files. They show that the 2-D Maxwell equations describing propagation of the transverse-magnetic mode in anisotropic media is mathematically equivalent to the SH wave equation in an anisotropic-viscoelastic solid where attenuation is described with the Maxwell model. Later, Carcione and Robinson [2002] establish the analogy for the reflection-transmission problem at a single interface, showing that contrasts in compressibility yields the reflection coefficient for light polarized perpendicular to the plane of incidence (Fresnel's sine law - the electric vector perpendicular to the plane of incidence), and density contrasts yields the reflection coefficient for light polarized in the plane of incidence (Fresnel's tangent law). These two papers provide the time-domain differentials equations and the analysis for a single interface, while in the present paper, we exploit the analogy for a layer embedded between two dissimilar half spaces, showing how the same equation hold for shear, compressional and electromagnetic waves, in addition to the quantum tunnel effect.

In 1821, Fresnel obtained the wave surface of an optically biaxial crystal, assuming that light waves are vibrations of the ether in which longitudinal vibrations (P waves) do not propagate. An anisotropic elastic medium having orthorhombic symmetry, mathematically analogous to Fresnel's crystal, exists. This medium was found by Carcione and Helbig [2008], who obtained the differential equations describing the wave motion.

In analytical terms, the above mentioned papers deal with a homogeneous medium and a single interface. The next step to exploit the analogy is to study the wave re- 
sponse of a layer, namely the reflection and transmission of waves by a thin plane layer embedded in a homogeneous medium. The reflection coefficient for isotropic media is given in Equation (5.18) of Brekhovskikh [1960] and Section 1.6.4. (Equation 57) of Born and Wolf [1964]. These authors report the expressions for the TE case. Here, we mainly consider the TM case. TE waves are equivalent to acoustic $(\mathrm{P})$ waves propagating in liquids, while TM waves are equivalent to shear $(\mathrm{SH})$ waves propagating in solids [Carcione and Robinson 2002]. Moreover, the equation in Born and Wolf [1964] is given for purely dielectric media but the lossy version can be obtained by replacing the real dielectric constant by the complex permittivity. An equivalent viscoelastic model can be obtained in the elastic case, using the Maxwell model. Here, we consider the media to be anisotropic with one of the principal axes parallel to the layering.

In geophysics, the problem finds application in reflection seismology. Widess [1973] and Bakke and Ursin [1998] consider the normal incidence case of a thin layer, while Liu and Schmitt [2003] obtain the reflection coefficient as a function of the incidence angle corresponding to P waves. Bradford and Deeds [2006] and Deparis and Garambois [2009] consider the electromagnetic case applied to amplitude variations with the incidence angle for surface radar problems. In quantum mechanics, the same mathematical approach can be used to analyze the tunnel effect [Anderson 1971].

We choose to present geophysical examples mainly. The analogy is applied to horizontally polarized shear $(\mathrm{SH})$ waves through a fluid-filled fracture and TM waves through thin metal films, which are shown to be similar to the tunnel effect. Moreover, other examples consider $\mathrm{P}$ and $\mathrm{SH}$ seismic waves reflected from a thin and lossy sandstone bed and TM waves reflected from a dyke of quartz. Finally, we compare the TM and TE cases by using the analogy.

\section{Reflection and transmission coefficients of a thin layer. Viscoelasticity}

We start from the viscoelastic equations and then apply the analogy to obtain the equivalent electromagnetic and quantum expressions. Figure 1 shows the layer embedded between two half spaces with different properties. In the following, we denote particle velocity by $v$, stress by $\sigma$, magnetic field by $H$, electric field by $E$, density by $\rho$, elasticity constant by $c$, viscosity by $\eta$, magnetic permeability by $\mu$, dielectric permittivity by $\epsilon$ and electrical conductivity by $\sigma$. Moreover, $(x, y, z)$ indicates the spatial variables, $\partial x$ a partial derivative with respect to $x$ and a dot above a variable denotes time differentiation. To distinguish between the stress and conductivity components, we use letters and numbers as

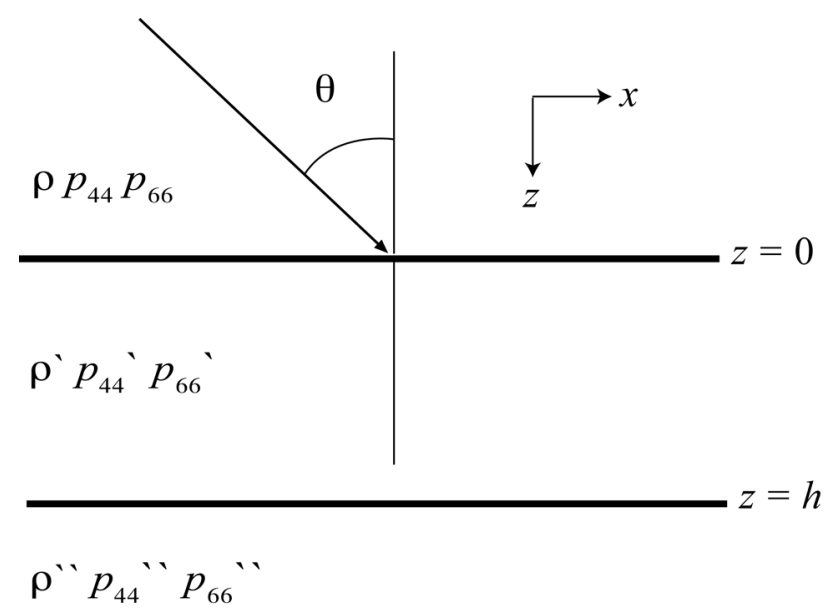

Figure 1. Plane wave propagating through a layer. The viscoelastic properties are indicated.

subindices, respectively, e.g., $\sigma_{x y}$ is a stress component and $\sigma_{11}$ is a conductivity component.

The viscoelastic medium is characterized by the mass density $\rho$ and elasticity and viscosity matrices

$$
\left(\begin{array}{cc}
c_{44} & 0 \\
0 & c_{66}
\end{array}\right) \text { and }\left(\begin{array}{cc}
\eta_{44} & 0 \\
0 & \eta_{66}
\end{array}\right)
$$

respectively [Carcione and Cavallini 1995, Carcione 2007]. It corresponds to a viscoelastic medium described by the Maxwell mechanical model. The subindices "44" and " 66 " refer to the Voigt notation of the elasticity and viscosity tensors [e.g., Carcione 2007].

The differential equations describing the wave motion of $\mathrm{SH}$ (shear) waves are

$$
\begin{gathered}
\partial_{x} \sigma_{x y}+\partial_{z} \sigma_{y z}=\rho \dot{v}_{y}, \\
-\partial_{z} v_{y}=-\eta_{44}^{-1} \sigma_{y z}-c_{44}^{-1} \dot{\sigma}_{y z}, \\
\partial_{x} v_{y}=\eta_{66}^{-1} \sigma_{x y}+c_{66}^{-1} \dot{\sigma}_{x y} .
\end{gathered}
$$

The boundary conditions at the interfaces require continuity of

$$
\sigma_{y z} \text { and } v_{y}
$$

[e.g., Carcione 2007]. In the electromagnetic case, continuity of the tangential components of the electric and magnetic fields is required [Born and Wolf 1964] (see below). Let us assume that the incident, reflected and refracted waves are identified by the subscripts and superscripts $I, R$ and $T$.

For a single interface, the particle velocities of the incident, reflected and refracted waves are given by

$$
\begin{gathered}
v_{y}^{I}=\exp \left[\mathrm{i} \omega\left(t-s_{x} x-s_{z} z\right)\right], \\
v_{y}^{R}=R \exp \left[\mathrm{i} \omega\left(t-s_{x} x-s_{z}^{R} z\right)\right], \\
v_{y}^{T}=T \exp \left[\mathrm{i} \omega\left(t-s_{x} x-s_{z}^{T} z\right)\right],
\end{gathered}
$$


respectively, where $\left(s_{x}, s_{z}\right)^{\top}$ is the slowness vector, $\omega$ is the angular frequency, $R$ and $T$ are the reflection and refraction (transmission) coefficients, $t$ is the time variable and $i=\sqrt{-1}$. The equations obtained below hold for an incident inhomogeneous plane waves (non-uniform waves in electromagnetism), i.e., waves for which the wavenumber and attenuation vectors do not point in the same direction. In the special case where these two vector coincide, the wave is termed homogeneous (uniform in electromagnetism), and we have

$$
\left(s_{x}, s_{z}\right)^{\top}=\frac{1}{v}(\sin \theta, \cos \theta)^{\top},
$$

where $\theta$ is the incidence angle and

$$
v=\sqrt{\frac{p_{66} \sin ^{2} \theta+p_{44} \cos ^{2} \theta}{\rho}}
$$

is the complex velocity, with

$p_{44}=\left(\frac{1}{c_{44}}+\frac{1}{\mathrm{i} \omega \eta_{44}}\right)^{-1}$ and $p_{66}=\left(\frac{1}{c_{66}}+\frac{1}{\mathrm{i} \omega \eta_{66}}\right)^{-1}$

[Carcione and Cavallini 1995, Carcione 2007].

In the general case, the reflection and transmission coefficients (TM case in electromagnetism) are given by

$$
r=\frac{Z_{I}-Z_{T}^{\prime}}{Z_{I}+Z_{T}^{\prime}}, \quad \tau=\frac{2 Z_{I}}{Z_{I}+Z_{T}^{\prime}},
$$

where

$$
Z_{I}=p_{44} s_{z}, \quad Z_{T}^{\prime}=p_{44}^{\prime} s_{z}^{T^{\prime}},
$$

with

$$
s_{x}^{R}=s_{x}^{T}=s_{x}^{I}=s_{x}(\text { Snell's law }), s_{z}^{R}=-s_{z}
$$

and

$$
s_{z}^{T^{\prime}}=\frac{1}{\sqrt{p_{44}^{\prime}}} \mathrm{pv} \sqrt{\rho^{\prime}-\rho_{66}^{\prime} s_{x}^{2}},
$$

with "pv" denoting the principal value [Carcione and Robinson 2002, Carcione 2007].

To obtain the reflection and transmission coefficients of the layer, we follow the procedure indicated in Section 6.4 of Carcione [2007]. At depth $z$ in the layer, the particle-velocity field is a superposition of upgoing and downgoing waves of the form

$$
\begin{aligned}
v_{y}(z)= & {\left[V^{-} \exp \left(i \omega s_{z}^{T^{\prime}} z\right)+\right.} \\
& \left.+V^{+} \exp \left(-i \omega s_{z}^{T^{\prime}} z\right)\right] \exp \left[i \omega\left(t-s_{x} x\right)\right],
\end{aligned}
$$

where $V^{-}$and $V^{+}$are upgoing- and downgoing-wave amplitudes. Combining Equations (3) and (12), the normal stress component is

$$
\begin{aligned}
\sigma_{y z}(z)= & \frac{p_{44}^{\prime}}{i \omega} \partial_{z} v_{y}=Z_{T}^{\prime}\left[V^{-} \exp \left(i \omega s_{z}^{T^{\prime}} z\right)-\right. \\
& \left.-V^{+} \exp \left(-i \omega s_{z}^{T^{\prime}} z\right)\right] \exp \left[i \omega\left(t-s_{x} x\right)\right] .
\end{aligned}
$$

Omitting the phase exp $\left[\mathrm{i} \omega\left(t-s_{\chi} x\right)\right]$, the particlevelocity/stress vector can be written as

$$
\begin{aligned}
& \mathbf{t}(z)=\left(\begin{array}{c}
v_{y} \\
\sigma_{y z}
\end{array}\right)= \\
& =\left(\begin{array}{cr}
\exp \left(i \omega s_{z}^{T^{\prime}} z\right) & \exp \left(-\mathrm{i} \omega s_{z}^{T^{\prime}} z\right) \\
Z_{T}^{\prime} \exp \left(i \omega s_{z}^{T^{\prime}} z\right)-Z_{T}^{\prime} \exp \left(-i \omega s_{z}^{T^{\prime}} z\right)
\end{array}\right)\left(\begin{array}{c}
V^{-} \\
V^{+}
\end{array}\right) \equiv \\
& \equiv \mathbf{T}(z)\left(\begin{array}{c}
V^{-} \\
V^{+}
\end{array}\right) .
\end{aligned}
$$

Then, the fields at $z=0$ and $z=h$ are related by the following equation:

$$
\mathbf{t}(0)=\mathbf{B} \cdot \mathbf{t}(h)
$$

where

$$
\begin{aligned}
& \mathbf{B}=\mathbf{T}(0) \cdot \mathbf{T}^{-1}(h)= \\
& \left(\begin{array}{cc}
\cos \varphi & -\mathrm{i}\left(Z_{T}^{\prime}\right)^{-1} \sin \varphi \\
-\mathrm{i} Z_{T}^{\prime} \sin \varphi & \cos \varphi
\end{array}\right), \varphi=\omega s_{z}^{T^{\prime} h}
\end{aligned}
$$

Equation (15) plays the role of a boundary condition. Note that when $h=0, \mathbf{B}$ is the identity matrix.

Using Equations (2), (4) and (9), the particle-velocity/stress field at $z=0$ and $z=h$ can be expressed as

$$
\begin{aligned}
& \mathbf{t}(0)=\left(1+R, Z_{I}(R-1)\right)^{\top}, \\
& \mathbf{t}(h)=T\left(1,-Z_{T}^{\prime \prime}\right)^{\top},
\end{aligned}
$$

where

$$
s_{z}^{T^{\prime \prime}}=\frac{1}{\sqrt{p_{44}^{\prime \prime}}} \mathrm{pv} \sqrt{\rho^{\prime \prime}-\rho_{66}^{\prime \prime} s_{x}^{2}}, \quad Z_{T}^{\prime \prime}=p_{44}^{\prime \prime} s_{z}^{T^{\prime \prime}},
$$

and $R$ and $T$ are here the reflection and transmission coefficients of the layer.

Substituting Equation (17) into (16), we have

$R=\frac{A-1}{A+1}, \quad A=\left(\frac{Z_{I}}{Z_{T}^{\prime}}\right) \frac{Z_{T}^{\prime} \cos \varphi+\mathrm{i} Z_{T}^{\prime \prime} \sin \varphi}{Z_{T}^{\prime \prime} \cos \varphi+\mathrm{i} Z_{T}^{\prime} \sin \varphi}$.

If $h=0, \varphi=0$ and we obtain $R=\left(Z_{I}-Z_{T}^{\prime \prime}\right) /\left(Z_{I}+\right.$ $\left.Z_{T}^{\prime \prime}\right)$ as expected. If, moreover, the two media have the same properties, it is $Z_{T}^{\prime \prime}=Z_{I}$ and the reflection coefficient is nil. Equation (19) coincides with Equation (2) of Bradford and Deeds [2006] in the absence of wave loss. These authors used the convention $\exp (-\mathrm{i} \omega t)$, instead of $\exp (\mathrm{i} \omega t)$ used here, so that the sign of the frequency has to be changed in Equation (2).

If the upper and lower half spaces have the same properties, we get

$$
R=\frac{r[1-\exp (-2 \mathrm{i} \varphi)]}{1-r^{2} \exp (-2 \mathrm{i} \varphi)},
$$


where $r$ is given by Equation (8).

Equation (19) is similar to Equation (5.22) of Born and Wolf [1964] if the media are isotropic and lossless. In order to be identical, this equation has to be rewritten as

$$
R=\frac{r_{12}+r_{23} \exp (-2 \mathrm{i} \varphi)}{1+r_{12} r_{23} \exp (-2 \mathrm{i} \varphi)},
$$

where

$$
r_{12}=r \quad \text { and } \quad r_{23}=\frac{Z_{T}^{\prime}-Z_{T}^{\prime \prime}}{Z_{T}^{\prime}+Z_{T}^{\prime \prime}}
$$

Equation (21) differs from Equation (5.22) in the fact that $r_{12}$ and $r_{23}$ correspond here to TM waves, while in Equation (5.22) these quantities correspond to TE waves. Another difference is the minus sign in the exponent, which arises from the fact that Born and Wolf [1964] use the factor $\exp (-i \omega t)$. In the case in which the media above and below the layer have the same properties, i.e., when $r_{23}=-r_{12}=-r$, Equation (22) becomes Equation (20).

On the other hand, the transmission coefficient can be obtained from Equation (17). It gives

$T=2\left[\left(1+\frac{Z_{T}^{\prime \prime}}{Z_{I}}\right) \cos \varphi+i\left(\frac{Z_{T}^{\prime \prime}}{Z_{T}^{\prime}}+\frac{Z_{T}^{\prime}}{Z_{I}}\right) \sin \varphi\right]^{-1}$,

which can be written as

$$
\begin{gathered}
T=\frac{\tau_{12} \tau_{23} \exp (-\mathrm{i} \varphi)}{1+r_{12} r_{23} \exp (-2 \mathrm{i} \varphi)}, \\
\tau_{12}=\tau \quad \text { and } \quad \tau_{23}=\frac{2 Z_{T}^{\prime}}{Z_{T}^{\prime}+Z_{T}^{\prime \prime}} .
\end{gathered}
$$

This expression coincides in form with that of Born and Wolf ([1964], Equation 58).

\section{Analogy with a modified acoustic wave equation}

Carcione and Robinson [2002] introduced a modified acoustic wave equation with a time-dependent mass density. Denoting the particle velocities by $v$ and the pressure field by $p$, the equations can be written as

$$
\begin{aligned}
& \partial_{z} v_{z}+\partial_{x} v_{x}=-\kappa \dot{p}, \\
& -\partial_{z} p=\gamma v_{z}+\rho_{f} \dot{v}_{z}, \\
& -\partial_{x} p=\gamma v_{x}+\rho_{f} \dot{v}_{x},
\end{aligned}
$$

where $\kappa$ is the fluid compressibility, $\rho_{f}$ is the fluid density and $\gamma=0$ yields the standard acoustic equations of motion. Equations (26) correspond to a generalized density of the form $\gamma I(t)+\rho_{f} H(t)$, where $H(t)$ is the Heaviside function and $I(t)$ is the integral operator. In the frequency domain, a complex density governs the wave equation and has the expression $\rho_{f}+\gamma /(\mathrm{i} \omega)$. This additional density term introduces wave loss as that of the Biot attenuation mechanism in poroelasticity theory [e.g., Carcione 2007].

Equations (26) are mathematically analogous to the isotropic-medium SH Equations (2) for the following correspondence

$$
\begin{aligned}
\sigma_{x y} & \Longleftrightarrow v_{x} \\
\sigma_{y z} & \Longleftrightarrow v_{z} \\
v_{y} & \Longleftrightarrow-p \\
\eta^{-1} & \Longleftrightarrow \gamma \\
c^{-1} & \Longleftrightarrow \rho_{f} \\
\rho & \Longleftrightarrow \kappa
\end{aligned}
$$

where $c_{44}=c_{66}=c$ and $\eta_{44}=\eta_{66}=\eta$.

\section{Analogy with electromagnetism}

\subsection{SH-TM analogy}

The analogy between SH waves and transversemagnetic (TM) waves states that the equivalent electromagnetic medium has the following permittivity and conductivity tensors

$$
\left(\begin{array}{ccc}
\epsilon_{11} & 0 & 0 \\
0 & \epsilon_{11} & 0 \\
0 & 0 & \epsilon_{33}
\end{array}\right) \text { and }\left(\begin{array}{ccc}
\sigma_{11} & 0 & 0 \\
0 & \sigma_{11} & 0 \\
0 & 0 & \sigma_{33}
\end{array}\right) \text {, }
$$

respectively, describing a uniaxial absorbing crystal.

The TM Maxwell's equations are

$$
\begin{gathered}
\partial_{x} E_{z}-\partial_{z} E_{x}=\mu \dot{H}_{y}, \\
-\partial_{z} H_{y}=\sigma_{11} E_{x}+\epsilon_{11} \dot{E}_{x}, \\
\partial_{x} H_{y}=\sigma_{33} E_{z}+\epsilon_{33} \dot{E}_{z} .
\end{gathered}
$$

The mathematical analogy is

$$
\begin{aligned}
\left(\begin{array}{c}
v_{y} \\
\sigma_{y z} \\
\sigma_{x y}
\end{array}\right) & \Longleftrightarrow\left(\begin{array}{c}
H_{y} \\
-E_{x} \\
E_{z}
\end{array}\right) \\
\left(\begin{array}{cc}
c_{44}^{-1} & 0 \\
0 & c_{66}^{-1}
\end{array}\right) & \Longleftrightarrow\left(\begin{array}{cc}
\epsilon_{11} & 0 \\
0 & \epsilon_{33}
\end{array}\right) \\
\left(\begin{array}{cc}
\eta_{44}^{-1} & 0 \\
0 & \eta_{66}^{-1}
\end{array}\right) & \Longleftrightarrow\left(\begin{array}{cc}
\sigma_{11} & 0 \\
0 & \sigma_{33}
\end{array}\right) \\
\rho & \Longleftrightarrow \mu,
\end{aligned}
$$

[Carcione and Cavallini 1995, Carcione 2007], where the electromagnetic tensors are redefined here as $2 \times 2$ matrices for simplicity. 


\subsection{SH-TE analogy}

The TE Maxwell equations are

$$
\begin{aligned}
\partial_{z} H_{x}-\partial_{x} H_{z} & =\varepsilon_{22} \dot{E}_{y}, \\
\partial_{z} E_{y} & =\mu \dot{H}_{x}, \\
-\partial_{x} E_{y} & =\mu \dot{H}_{z}
\end{aligned}
$$

[Carcione 1998, Carcione and Robinson 2002], where we have considered the lossless case (zero conductivity). As can be seen, these equations are similar to those of an isotropic medium, since only one component of the permittivity tensors appears. This fact indicates that TE waves are also equivalent to sound waves.

The TM-TE analogy has been given by Carcione [1998] and Carcione and Robinson [2002]. The complete mathematical analogy is

\begin{tabular}{cll} 
TM & SH & TE \\
\hline$H_{y} \Longleftrightarrow v_{y}$ & $\Longleftrightarrow-E_{y}$ \\
$E_{x} \Longleftrightarrow-\sigma_{y z}$ & $\Longleftrightarrow H_{x}$ \\
$E_{z} \Longleftrightarrow \sigma_{x y}$ & $\Longleftrightarrow H_{z}$ \\
$\epsilon_{11} \Longleftrightarrow c_{44}^{-1}$ & $\Longleftrightarrow \mu$ \\
$\epsilon_{33} \Longleftrightarrow c_{66}^{-1}$ & $\Longleftrightarrow \mu$ \\
$\mu \Longleftrightarrow \rho$ & $\Longleftrightarrow \epsilon_{22}$
\end{tabular}

\section{Analogy with quantum mechanics. Tunnel effect}

For simplicity, we consider the time-independent Schrödinger equation

$$
-\frac{\hbar^{2}}{2 m} \Delta \phi=(E-V) \phi
$$

where $\hbar=1.055 \times 10^{-34} \mathrm{~J}$ s is Planck's constant, $m$ is the mass of the particle, $\phi$ is the wave function, $E$ is the total energy of the particle, $V$ is the height of the potential barrier, and $\Delta$ is the Laplacian.

On the other hand, in the isotropic case, Equation (2) can be written as

$$
\Delta v_{y}+\omega^{2} s^{2} v_{y}=0, \quad s=\sqrt{\frac{\rho}{p}},
$$

where $p=p_{44}=p_{66}$ and we have assumed an harmonic wave.

Equations (36) and (37) are mathematically equivalent if $\phi=v_{y}$ and

$$
s=\frac{\sqrt{2 m E}}{\hbar \omega} \text { and } s^{\prime}=\frac{\sqrt{2 m(E-V)}}{\hbar \omega}
$$

outside and inside the barrier, respectively. The tunnel effect occurs when $E<V$. In this case, the waves within the potential barrier are evanescent with a complex velocity given by

$$
v^{\prime}=\left(s^{\prime}\right)^{-1}=-\frac{i \omega \hbar}{\sqrt{2 m(V-\hbar \omega)}},
$$

where we have expressed the energy as $E=\hbar \omega$.

The analogy holds with $\mathrm{SH}$ waves at normal incidence $(\theta=0)$. Of interest is the transmission coefficient through the barrier, given by Equation (24), when the media above and below the layer (i.e., the barrier) have the same properties, i.e., when $r_{23}=-r_{12}=-r, \tau_{12}=\tau$ and $\tau_{23}=\left(Z_{T}^{\prime} / Z_{I}\right) \tau$. Then

$$
T=\frac{\tau^{2}\left(Z_{T}^{\prime} / Z_{I}\right) \exp (-\mathrm{i} \varphi)}{1-r^{2} \exp (-2 \mathrm{i} \varphi)},
$$

where $r$ and $\tau$ are given by Equation (8). At normal incidence,

$Z_{I}=\frac{\rho}{s}, Z_{T}^{\prime}=\frac{\rho}{s^{\prime}}$ and $\varphi=\omega s^{\prime} h=\frac{E}{\hbar} s^{\prime} h$

where the density $\rho$ is uniform in the whole space and can have any arbitrary value. Alternatively, the analogy can be established by assuming variable density media and uniform stiffness $p$. In this case, $\rho=s p$ and $\rho^{\prime}=s^{\prime} p$, with $Z_{I}=p s$ and $Z_{T}^{\prime}=p s^{\prime}$ with $p$ taking any arbitrary value.

Equation (40) coincides with Equations (5.11) (E> $V)$ and (5.12) $(E<V)$ of Anderson [1971], considering that this author reports $T^{2}$ instead of $T$.

The interpretation of the transmission coefficient $|T|^{2}$ in this case is the probability of a particle to be tunneling through the barrier. An approximation to Equation (40) can be obtained for $V>E$ and $\varphi \gg 1$,

$$
\begin{aligned}
& T \approx 4 \sqrt{\frac{E}{V}\left(1-\frac{E}{V}\right)} \exp (-\varphi) \propto \exp (-\varphi)= \\
&=\exp \left(-\frac{\sqrt{2 m(V-E)}}{\hbar} h\right),
\end{aligned}
$$

which is the simplified equation found in many textbooks [Feynman et al. 1965].

\section{Results}

We first consider cases equivalent to the tunnel effect. Particular cases of interest are fractures in elastic media and thin metal films in electromagnetic media. Assuming $c_{44}^{\prime}=c_{66}^{\prime} \rightarrow \infty$ and $\eta_{44}^{\prime}=\eta_{66}^{\prime} \equiv \eta^{\prime}$, we obtain a Newtonian fluid from Equation (7),

$$
p_{44}^{\prime}=p_{66}^{\prime}=p^{\prime}=\mathrm{i} \omega \eta^{\prime} \text {. }
$$

The complex velocity (6) becomes

$$
v=\sqrt{\frac{p^{\prime}}{\rho^{\prime}}}=\sqrt{\frac{i \omega \eta^{\prime}}{\rho^{\prime}}}=\sqrt{\frac{i \omega}{\gamma^{\prime} \kappa^{\prime}}},
$$

where the third and fourth expressions correspond to $\mathrm{SH}$ - and P-waves, respectively. 
On the other hand, an anisotropic metal has

$$
p_{44}^{\prime}=\frac{i \omega}{\sigma_{11}^{\prime}} \text { and } p_{66}^{\prime}=\frac{i \omega}{\sigma_{33}^{\prime}},
$$

with

$$
v=\sqrt{\frac{\mathrm{i} \omega}{\mu^{\prime}}\left(\frac{\sin ^{2} \theta}{\sigma_{33}^{\prime}}+\frac{\cos ^{2} \theta}{\sigma_{11}^{\prime}}\right)}=\sqrt{\frac{\mathrm{i} \omega}{\mu^{\prime} \sigma^{\prime}}},
$$

where the last expression holds for an isotropic medium, with $\sigma_{11}^{\prime}=\sigma_{33}^{\prime}=\sigma^{\prime}$.

Note that in the viscoelastic and electromagnetic cases $v \propto \sqrt{\mathrm{i}}$, while in quantum mechanics $v \propto \mathrm{i}$ (see Equation 39), i.e., there is a $\pi / 4$ phase difference.

Let us first consider SH waves and a fracture thickness $h=1 \mathrm{~cm}$. The upper and lower medium are the same and defined by $c_{44}=10 \mathrm{GPa}, c_{66}=14 \mathrm{GPa}$ and $\rho=2000 \mathrm{~kg} / \mathrm{m}^{3}$, with zero viscosity. Figure 2 shows the reflection coefficient of $\mathrm{SH}$ waves as a function of viscosity $\eta^{\prime}(\mathrm{a})$ (normal incidence, i.e., $\theta=0$ ) and incidence angle (b) (for $\eta^{\prime}=10 \mathrm{kPa} s$ ). The frequency is $f=\omega /(2 \pi)$ $=60 \mathrm{~Hz}$. As can be seen, increasing viscosity and inci-
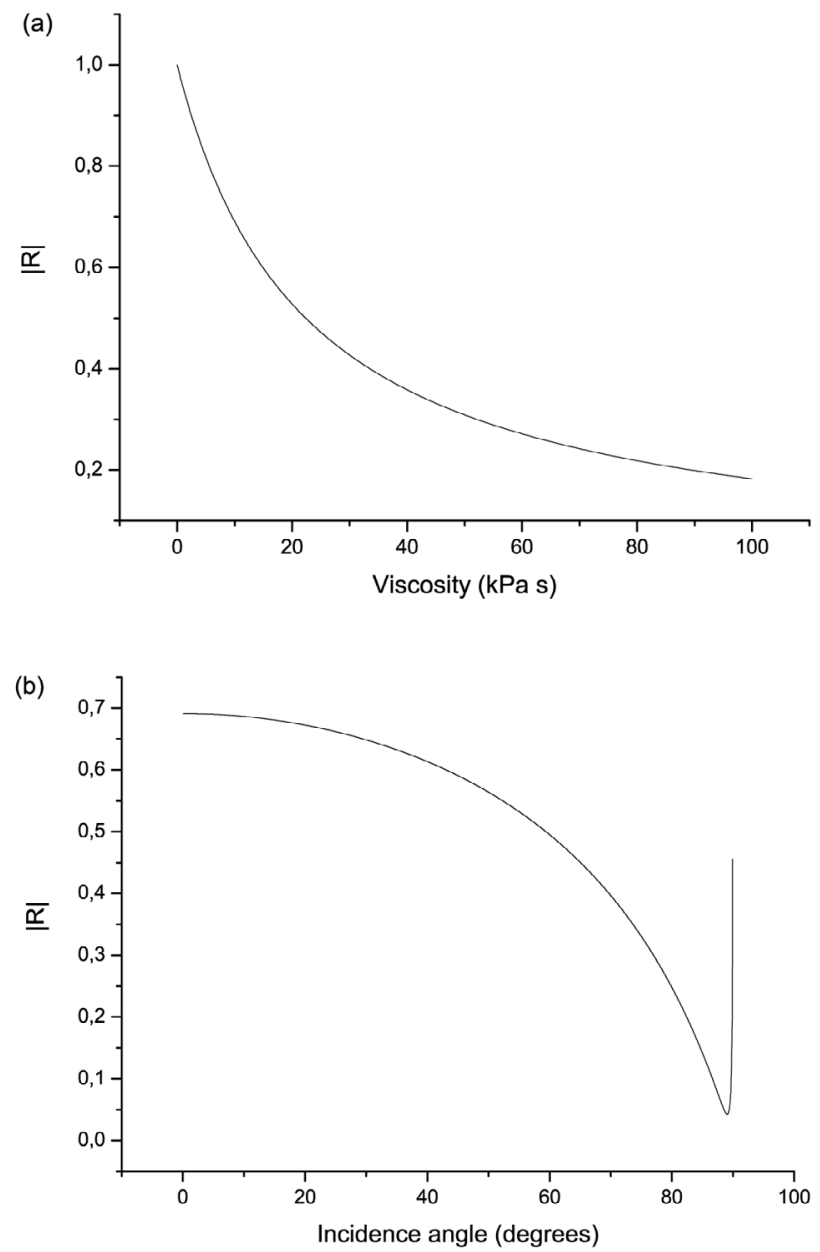

Figure 2. Absolute value of the reflection coefficient of $\mathrm{SH}$ waves as a function of viscosity (a) (normal incidence, i.e., $\theta=0$ ) and incidence angle (b) (for $\eta^{\prime}=10 \mathrm{kPa} \mathrm{s}$ ). The frequency is $f_{0}=60 \mathrm{~Hz}$ and the fracture thickness is $1 \mathrm{~cm}$. dence angle implies decreasing reflection amplitude and increasing transmission. For fluid viscosities comparable to water, oil or gas, the reflection coefficient is practically 1 for $h=1 \mathrm{~cm}$. To obtain a significant energy transmission the thickness has to be of the order of tens of Angstroms, since the wave has a shear nature whose polarization (particle motion) is parallel to the fracture surface. In real fractures, significant transmission takes place at larger thicknesses, since the surface of the fracture is rough and there are many contact points [e.g., Gangi and Carlson 1996].

Next, consider a thin conductive isotropic layer of thickness $h=1 \mu \mathrm{m}$ embedded in a lossless medium with permittivity components $\epsilon_{11}=8 \epsilon_{0}$ and $\epsilon_{33}=12 \epsilon_{0}$, where $\epsilon_{0}=8.8510^{-12} \mathrm{~F} / \mathrm{m}$. The magnetic permeability is the same for the two media, i.e., $\mu=\mu_{0}=4 \pi 10^{-7}$ $\mathrm{H} / \mathrm{m}$. Based on the analogy (30)-(33), Figure 3 shows the reflection coefficient of the electromagnetic wave as a function of conductivity (a) (normal incidence) and incidence angle (b) (for $\sigma^{\prime}=30000 \mathrm{~S} / \mathrm{m}$ ). The frequency is $1 \mathrm{MHz}$. As can be seen, the film totally re-
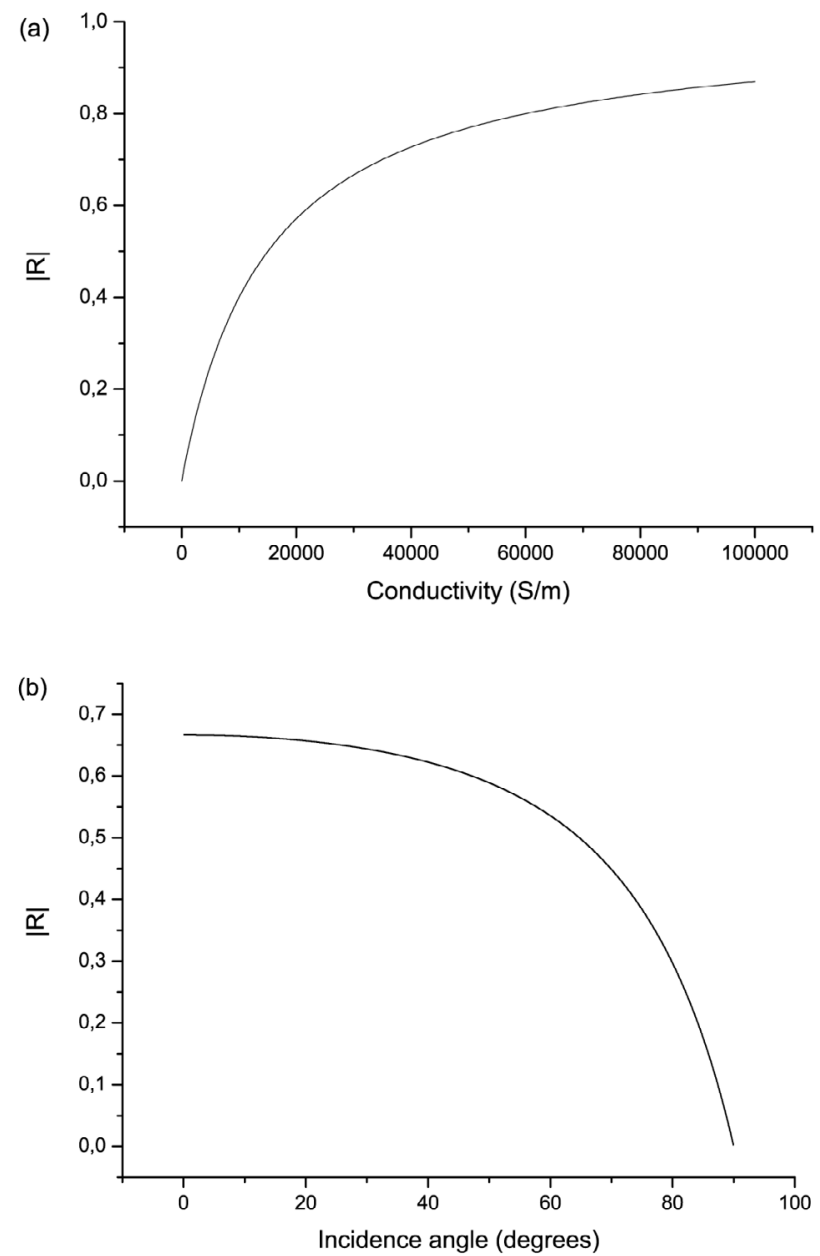

Figure 3. Absolute value of the reflection coefficient of the electromagnetic wave as a function of conductivity (a) (normal incidence) and incidence angle (b) (for $\sigma^{\prime}=30000 \mathrm{~S} / \mathrm{m}$ ). The frequency is $f_{0}=1 \mathrm{MHz}$ and the layer thickness is $h=1 \mu \mathrm{m}$. 


\begin{tabular}{cccccccccc}
\hline Medium & $\begin{array}{c}\epsilon_{s} \\
\left(\epsilon_{0}\right)\end{array}$ & $\begin{array}{c}\epsilon_{f} \\
\left(\epsilon_{0}\right)\end{array}$ & $\begin{array}{c}\sigma_{s} \\
(\mathrm{~S} / \mathrm{m})\end{array}$ & $\begin{array}{c}\sigma_{f} \\
(\mathrm{~S} / \mathrm{m})\end{array}$ & $\begin{array}{c}\epsilon \\
\left(\epsilon_{0}\right)\end{array}$ & $\begin{array}{c}K_{s} \\
(\mathrm{GPa})\end{array}$ & $\begin{array}{c}K_{f} \\
(\mathrm{GPa})\end{array}$ & $\begin{array}{c}\phi \\
(\%)\end{array}$ & $\begin{array}{c}K \\
(\mathrm{GPa})\end{array}$ \\
\hline Shale & 30 & 4.23 & 0.01 & 0.4 & $(30.15,-11.31)$ & 20 & 2.25 & 15 & 12.76 \\
Sandstone & 5 & 2 & 0 & $10^{-5}$ & $\left(3.79,-8.6 \times 10^{-4}\right)$ & 39 & 0.025 & 35 & 5.59 \\
\hline
\end{tabular}

Table 1. Properties. $A=3 ; \omega=100 \mathrm{MHz}$.

flects the wave for very high conductivities. This is the case for copper $\left(\sigma^{\prime}=610^{7} \mathrm{~S} / \mathrm{m}\right)$ for instance. Total transmission occurs for very resistive materials.

We consider now the tunnel effect. Let us define the reference wavelength

$$
\lambda=\frac{2 \pi \hbar}{\sqrt{2 m E}} .
$$

As an example, we consider the tunneling of an electron with mass $m=9.1 \times 10^{-31} \mathrm{~kg}$ and energy $E=$ $1.6 \times 10^{-19} \mathrm{~J}(1 \mathrm{eV})$, giving $\lambda=1.23 \mathrm{~nm}$. Figure $4 \mathrm{a}$ and $\mathrm{b}$ show the transmission coefficient as a function of $h$ for $V=10 E(a)$, and as a function of $V$ for $h=0.5 \mathrm{~nm}$ (b), normalized by $\lambda$ and $E$, respectively. As expected,
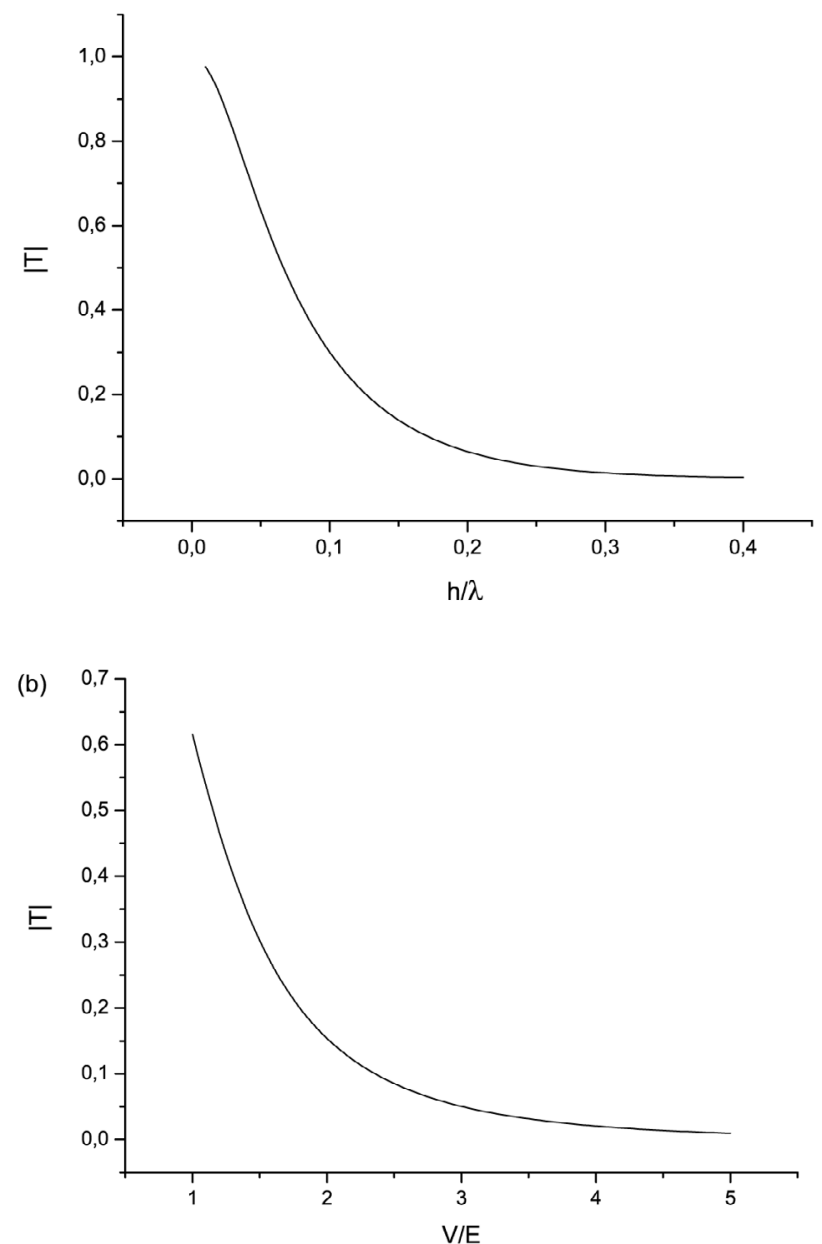

Figure 4. Absolute value of the transmission coefficient of an electron through a potential as a function of the width $h$ and height $V$ (properly normalized). In (a) $V=10 E$ and in (b) $h=0.5 \mathrm{~nm}$. the probability of tunneling decreases with increasing $h$ and $V$.

The next example considers a sandstone layer embedded in a shale formation, whose properties are taken from Thomsen [1986] (Table 1). The sandstone (Taylor sandstone) has the properties $v_{P}=\sqrt{c_{33} / \rho}=$ $3368 \mathrm{~m} / \mathrm{s}, v_{S}=\sqrt{c_{44} / \rho}=1829 \mathrm{~m} / \mathrm{s}, \gamma=\left(c_{66}-c_{44}\right) /\left(2 c_{44}\right)$ $=0.255$, and $\rho=2500 \mathrm{Kg} / \mathrm{m}^{3}$, where $v_{P}$ and $v_{S}$ are Pand Swave velocities and $c_{33}$ is an elasticity constant. The properties of the shale (Mesaverde mudshale) are $v_{P}=4529 \mathrm{~m} / \mathrm{s}, v_{S}=2703 \mathrm{~m} / \mathrm{s}, \gamma=0.046$, and $\rho=2520$ $\mathrm{Kg} / \mathrm{m}^{3}$. We obtain $c_{44}=18.4 \mathrm{GPa}, c_{66}=20.1 \mathrm{GPa}, c_{44}^{\prime}=$ $8.4 \mathrm{GPa}, c_{66}^{\prime}=12.6 \mathrm{GPa}$, and assume $\eta_{44}^{\prime}=\eta_{66}^{\prime}=0.5$ GPa s. This choice of the sandstone viscosity yields the
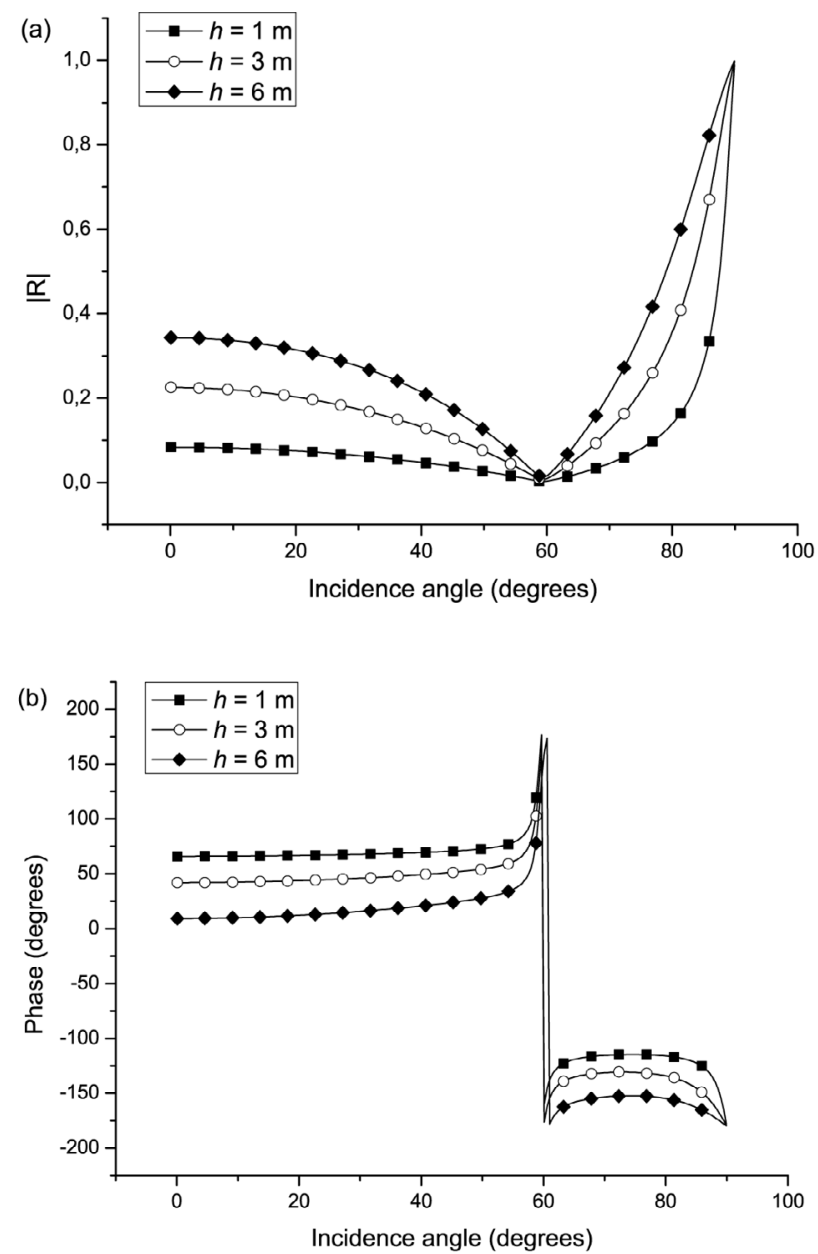

Figure 5. Sandstone layer in mudshale. Absolute value (a) and phase (b) of the $\mathrm{SH}$-wave reflection coefficient as a function of the incidence angle and three values of $h$. 
quality factors $Q_{44}=\omega \eta^{\prime} / c_{44}^{\prime}=9$ and $Q_{66}=\omega \eta^{\prime} / c_{66}^{\prime}=$ 6 (see Carcione [2007]: Equation 2.156), which implies high attenuation. In fact, the effective pressure for this sandstone is zero in Table 1 of Thomsen [1986] meaning that the medium is over-pressured. We consider a frequency $f=60 \mathrm{~Hz}$, which implies a wavelength of 30 $\mathrm{m}$ based on the $\mathrm{S}$-wave velocity of the layer. Figure 5 shows the reflection coefficient of the $\mathrm{SH}$ waves, Equation (20): absolute value (a) and phase (b), as a function of the incidence angle and three values of $h(1 \mathrm{~m}, 3 \mathrm{~m}$ and $6 \mathrm{~m}$ ). There is a Brewster angle at approximately $60^{\circ}$, where the reflection coefficients become almost zero and change phase [Carcione 2007].

We may obtain the reflection coefficient of $\mathrm{P}$ waves using the analogy (27). Then, we can use Equation (20) by replacing $c_{44}=c_{66}$ by $\rho_{f}^{-1}, \rho$ by $c_{33}^{-1}, c_{44}^{\prime}=c_{66}^{\prime}$ by $\left(\rho_{f}^{\prime}\right)^{-1}, \rho^{\prime}$ by $\left(c_{33}^{\prime}\right)^{-1}$, where $\rho_{f}$ and $\rho_{f}^{\prime}$ are the densities of the shale and sandstone, respectively. The viscosities are equal to $\infty$, i.e, there is no attenuation in this case. Figure 6 shows the absolute value (a) and phase (b) as a function of the incidence angle and three values
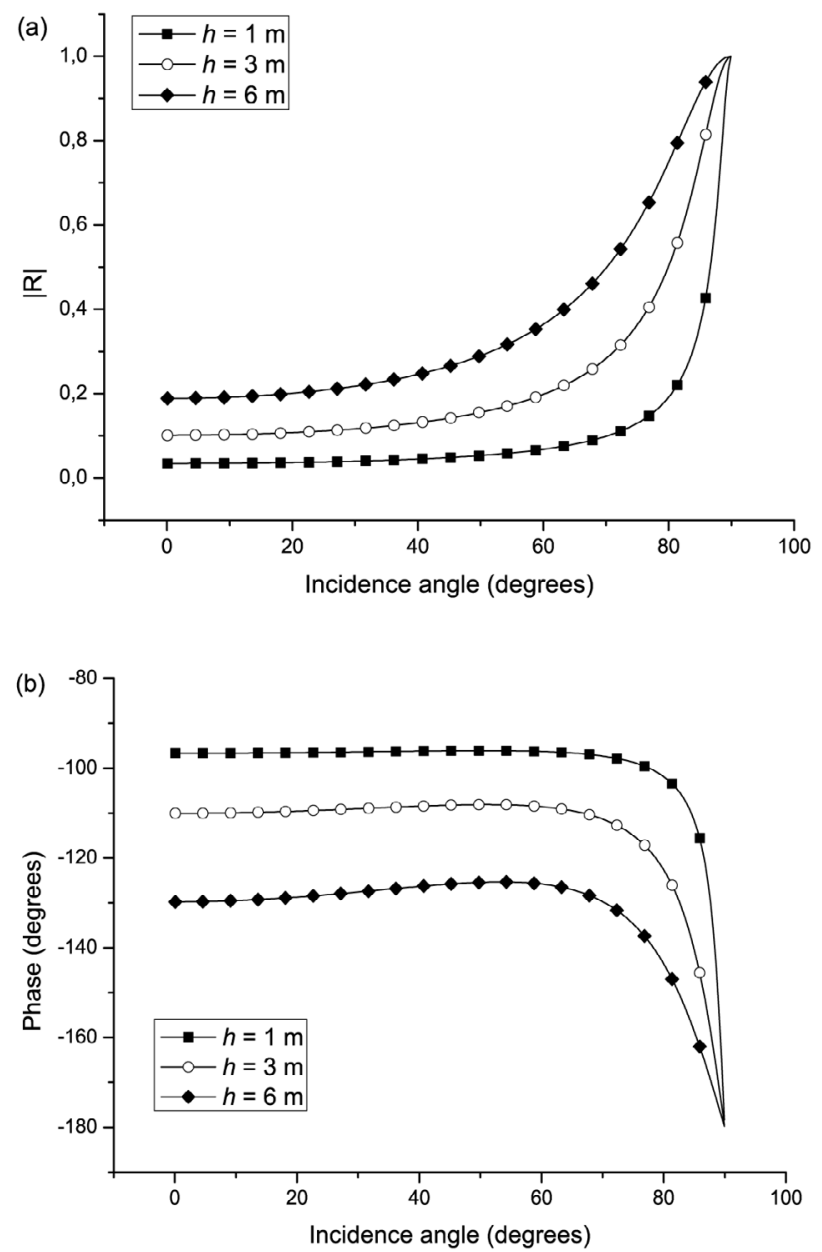

Figure 6. Sandstone layer in mudshale. Absolute value (a) and phase (b) of the P-wave reflection coefficient as a function of the incidence angle and three values of $h$. of $h(1 \mathrm{~m}, 3 \mathrm{~m}$ and $6 \mathrm{~m})$. In this case, the behaviour of the curves is quite different compared to S-wave case, with no Brewster angle.

Next, we compute the reflection coefficient of TM waves from a vein of quartz embedded in a limestone. We consider $\epsilon_{11}=9 \epsilon_{0}, \epsilon_{33}=0.8 \epsilon_{11}, \epsilon_{11}^{\prime}=\epsilon_{33}^{\prime}=$ $4 \epsilon_{0}, \mu=\mu_{0}$ and zero electrical conductivity for both rocks. The frequency is $f=100 \mathrm{MHz}$, typical of groundpenetrating radar surveys. The analogy (30)-(33) and Equation (20) are used to obtain the reflection coefficient, shown in Figure 7 for three different thicknesses of the quartz layer. In this case, there is a Brewster angle at $37^{\circ}$ approximately. The curves are similar to those of the $S$ wave, since it is known that shear and TM waves are analogous mathematically [e.g., Carcione and Cavallini 1995].

In the following, we test the SH-TE analogy and compare the TM and TE reflection coefficients. We consider the last example, but in the isotropic case, with $\mu=\mu_{0}, \epsilon_{11}=\epsilon_{22}=\epsilon_{33}=9 \epsilon_{0}$ and $\epsilon_{11}^{\prime}=\epsilon_{22}^{\prime}=\epsilon_{33}^{\prime}=4 \epsilon_{0}$. The thickness of the layer is $h=20 \mathrm{~cm}$. The TE curve
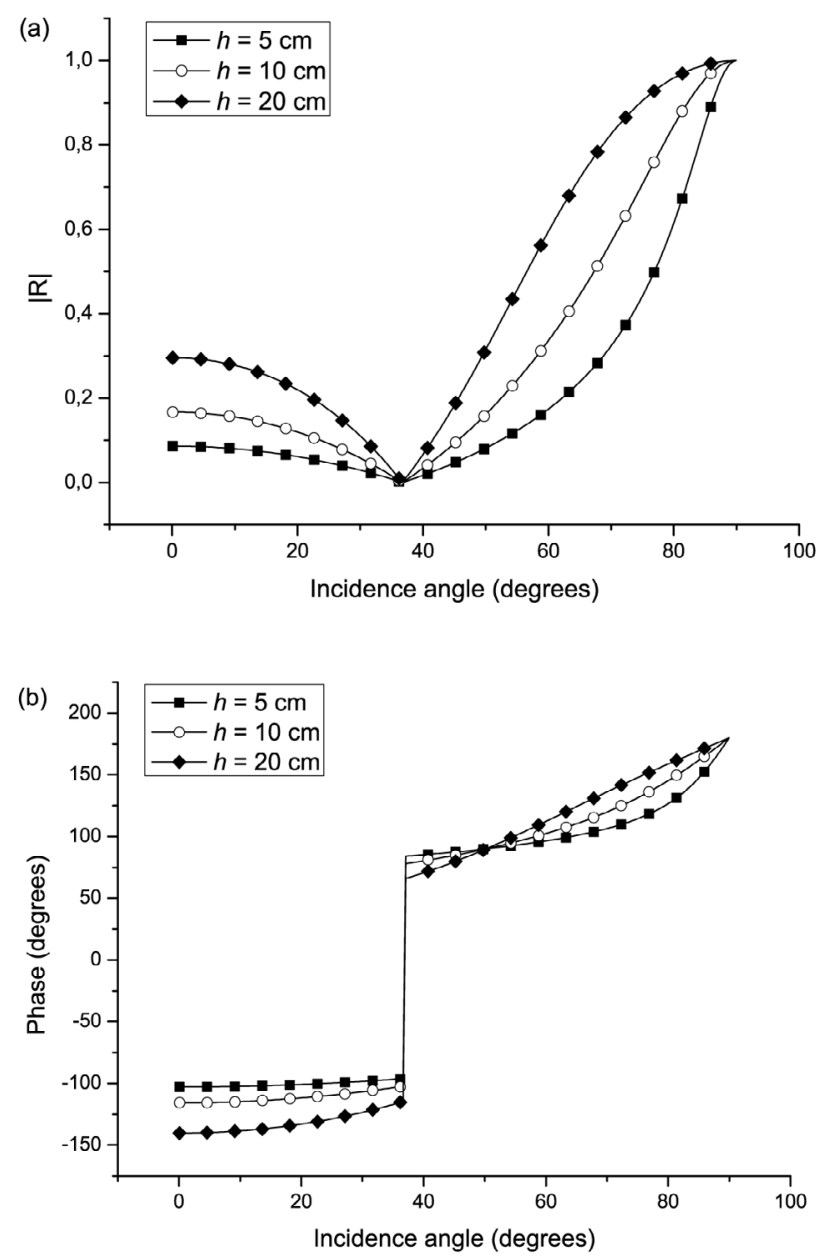

Figure 7. Vein of quartz in limestone. Absolute value (a) and phase (b) of the TM-wave reflection coefficient as a function of the incidence angle and three values of $h$. 
is in agreement with Equation (1) of Bradford and Deeds [2006]. It also agrees with Equation (2) of Deparis and Garambois [2009] provided that, in this paper, $\phi=2 d k_{2} \cos \theta_{i}$ is replaced by $\phi=2 d k_{2} \cos \theta_{t}$, which is the correct expression. The TE and TM curves are very different, mainly at the Brewster angle.

Finally, we consider a $\mathrm{CO}_{2}$-saturated sandstone embedded in brine-saturated shale and use a crossproperty relation to obtain the bulk modulus as a function of the complex permittivity [Carcione et al. 2007, Mavko et al. 2009]. The gas is in its supercritical state. In the following, $K, \rho, \sigma$ and $\epsilon$ denote bulk modulus, mass density, electrical conductivity and dielectric permittivity, respectively, and the subindices " $s$ " and " $f$ " denote grain and fluid properties. Gassmann equation gives the bulk modulus of the saturated rocks

$$
K=\frac{K_{s}-K_{m}(\phi)+\phi K_{m}(\phi)\left(K_{s} / K_{f}-1\right)}{1-\phi-K_{m}(\phi) / K_{s}+\phi K_{s} / K_{f}},
$$

where $\quad K_{m}(\phi)=K_{s}(1-\phi)^{(1-\phi+A) /(1-\phi)}$
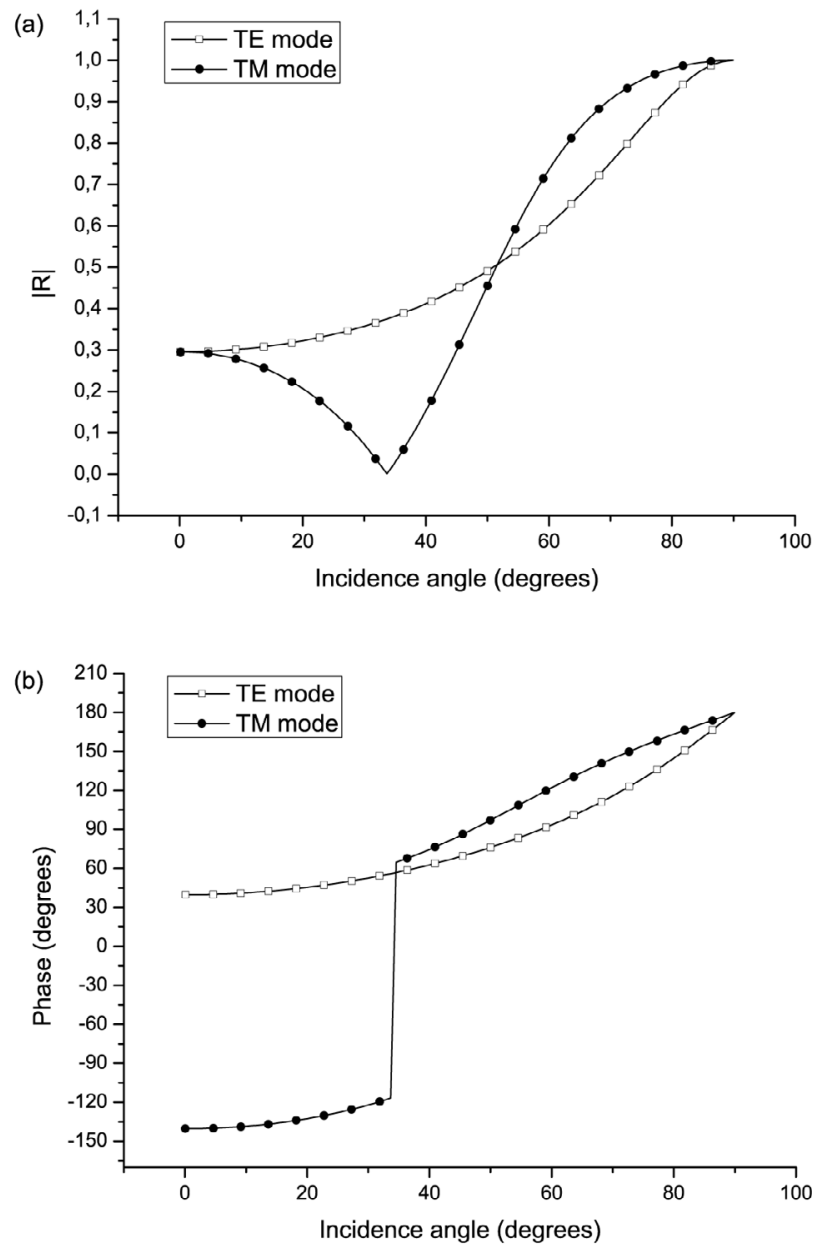

Figure 8. Absolute value (a) and phase (b) of the TE- and TM-wave reflection coefficients as a function of the incidence angle at $f=100$ $\mathrm{MHz}$ and $h=20 \mathrm{~cm}$.

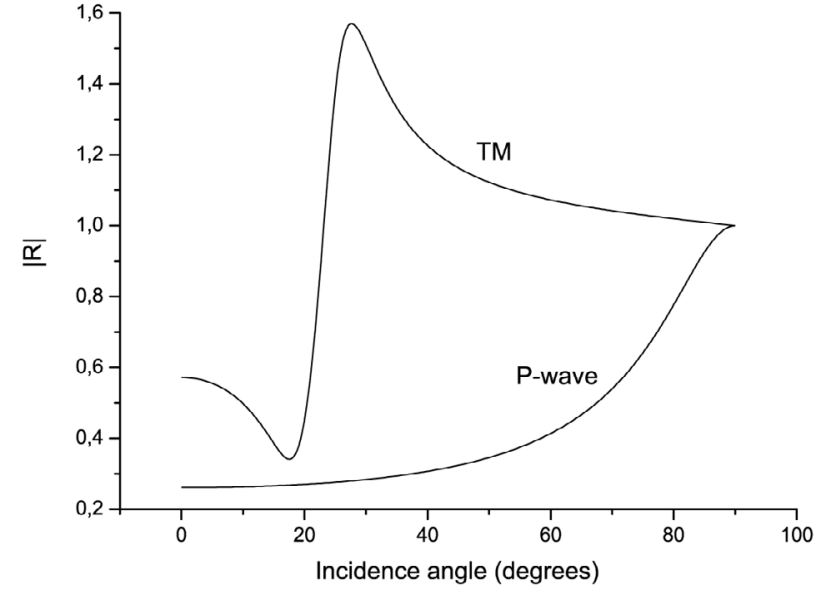

Figure 9. Absolute value of the TM-wave and P-wave reflection coefficients as a function of the incidence angle, corresponding to a sandstone layer saturated with supercritical gas embedded in brinesaturated shale.

is the dry-rock modulus and $\phi$ is the porosity, which can be obtained from the CRIM equation,

$$
\phi \approx \operatorname{Re}\left(\frac{\sqrt{\epsilon}-\sqrt{\epsilon_{s}-i \sigma_{s} / \omega}}{\sqrt{\epsilon_{f}-i \sigma_{f} / \omega}-\sqrt{\epsilon_{s}-i \sigma_{s} / \omega}}\right)
$$

where $\epsilon$ is the rock complex dielectric constant, $A$ is an empirical parameter and "Re" takes the real part. On the other hand, the rock density is given by $\rho=(1-\phi) \rho_{s}+$ $\phi \rho_{f}$. Assuming $\rho_{s}=2650 \mathrm{~kg} / \mathrm{m}^{3}$ for both shale and sandstone, $\rho_{f}=1000 \mathrm{~kg} / \mathrm{m}^{3}$ for brine and $\rho_{f}=500 \mathrm{~kg} / \mathrm{m}^{3}$ for $\mathrm{CO}_{2}$, we obtain $\rho=2400 \mathrm{~kg} / \mathrm{m}^{3}$ and $\rho=1897 \mathrm{~kg} / \mathrm{m}^{3}$, respectively. Moreover, we assume the properties given in Table 1, which also shows the calculated Gassmann moduli. We compute the P-wave and TM reflection coefficients for a wavelength/thickness ratio $r=10$, i.e., $\omega h=2 \pi c / r$, where $c$ is the P-wave velocity or the electromagnetic TM-wave velocity $(100 \mathrm{MHz})$ of the layer. Figure 9 shows the absolute value of the reflection coefficients versus incidence angle. As can be seen, the results indicate that the layer is more detectable with (high-frequency) electromagnetic methods.

\section{Conclusions}

Theories describing wave phenomena - and diffusion - in different fields of physics consist in partial differential equations, which have identical or similar mathematical expressions. Here, we have considered the reflection/transmission problem through an anisotropic and lossy layer. The equations hold for any layer thickness, not necessarily thin, although their uses are relevant for thin layers, i.e., when the wavelength of the signal is much larger than the thickness. We have shown that the same mathematical equations can be used in elastodynamics, electromagnetism and quantum mechanics by establishing appropriate analogies 
between the different physical variables and medium properties. In particular, the analogy has been obtained for $\mathrm{P}$ and SH elastic waves, TE and TM electromagnetic waves and wave mechanics in quantum theory.

Further research involves the analysis of the present problem in the space-time domain using numerical simulations. In this case, the same computer code, with appropriate input variables can be used to solve the different physical problems.

\section{References}

Anderson, E. (1971). Modern Physics and quantum mechanics, W.B. Saunders Co.

Bakke, N.E., and B. Ursin (1998). Thin-bed AVO effects, Geophysical Prospecting, 46, 571-587.

Born, M., and E. Wolf (1964). Principles of Optics, Oxford: Pergamon Press.

Bradford, J.H., and J.C. Deeds (2006). Ground-penetrating radar theory and application of thin-bed offset-dependent reflectivity, Geophysics, 71, K47-K57.

Brekhovskikh, L.M. (1960). Waves in layered media, Academic Press Inc.

Carcione, J.M., and F. Cavallini (1995). On the acousticelectromagnetic analogy, Wave Motion, 21, 149-162.

Carcione, J.M. (1998). Radiation patterns for GPR forward modeling, Geophysics, 63, 424-430.

Carcione, J.M., and E. Robinson (2002). On the acousticelectromagnetic analogy for the reflection-refraction problem, Studia Geophysica et Geodaetica, 46, 321-345.

Carcione, J.M. (2007). Wave Fields in Real Media. Theory and numerical simulation of wave propagation in anisotropic, anelastic, porous and electromagnetic media, 2nd edition, Elsevier.

Carcione, J.M., B. Ursin and J.I. Nordskag (2007). Crossproperty relations between electrical conductivity and the seismic velocity of rocks, Geophysics, 72, E193-E204.

Carcione, J.M., and K. Helbig (2008). Elastic medium equivalent to Fresnels double refraction crystal, J. Acoust. Soc. Am., 124 (4), 2053-2060.

Deparis, J., and S. Garambois (2009). On the use of dispersive APVO GPR curves for thin-bed properties estimation: Theory and application to fracture characterization, Geophysics, 74, J1-J12.

Feynman, R.P., R.B. Leighton and M. Sands (1965). The Feynman lectures on physics 13, Addison-Wesley. ISBN 0-7382-0008-5.

Gangi, A.F., and R.L. Carlson (1996). An asperity-deformation model for effective pressure, Tectonophysics, 256, 241-251.

Liu, L., and D.R. Schmitt (2003). Amplitude and AVO responses of a single thin bed, Geophysics, 68, 1161-1168.
Mavko, G., T. Mukerji and J. Dvorkin (2009). The rock physics handbook: tools for seismic analysis in porous media, Cambridge Univ. Press.

Thomsen, L. (1986). Weak elastic anisotropy, Geophysics, 51, 1954-1966.

Tonti, E. (1972). On the mathematical structure of a large class of physical theories, Accademia Nazionale dei Lincei, excerpt from 'Rendiconti della Classe di Scienze fisiche, matematiche e naturali', Series 8, vol. 52 (1).

Tonti, E. (1976). The reason for analogies between physical theories, Appl. Math. Modelling, 1, 37-50.

Widess, M.B. (1973). How Thin is a Thin Bed?, Geophysics, 38, 1176-1180.

${ }^{\star}$ Corresponding author: José M. Carcione, Istituto Nazionale di Oceanografia e di Geofisica Sperimentale (OGS), Sgonico (Trieste), Italy; email: jcarcione@inogs.it.

C 2014 by the Istituto Nazionale di Geofisica e Vulcanologia. All rights reserved. 